\title{
Comparison of Two Phenotypic Double Disc Diffusion Test (DDDT) for Detection of Extended Spectrum $\beta$-Lactamase Production by Enterobacteriaceae and Pseudomonas Species at a Tertiary Care Hospital in Bangladesh
}

\author{
Taslima Yesmin ${ }^{1, *}$, Mohammed Abdun Nur Sayam ${ }^{2}$, Md. Manjurul Quader ${ }^{3}$, Abul Hossain Khan ${ }^{4}$, \\ Md. Abdullah Yusuf ${ }^{5}$ \\ ${ }^{1}$ Department of Microbiology, North Bengal Medical College, Bangladesh \\ ${ }^{2}$ Narayngonj General Hospital, Bangladesh \\ ${ }^{3}$ Department of Forensic Medicine, Sirajul Islam Medical College, Bangladesh \\ ${ }^{4}$ Department of Microbiology, TMSS Medical College, Bangladesh \\ ${ }^{5}$ Department of Microbiology, National Institute of Neurosciences \& Hospital, Bangladesh
}

Copyright (C) 2015 Horizon Research Publishing All rights reserved.

\begin{abstract}
Background: The detection of extended-spectrum $\beta$-lactamase-producing (ESBL) bacteria is of importance for infection control and epidemiological surveillance. Objective: The purpose of the present study was to compare two phenotypic methods for the detection of ESBL-positive Enterobacteriaceae and Pseudomonas species. Methodology: This cross sectional study was carried out in the Department of Microbiology at Mymensingh Medical College, Bangladesh from January 2011 to June 2011. Patients of all ages and genders presented with UTI or wound infection were taken as study population. Gram negative bacilli (GNB) were analyzed by two methods used for routine susceptibility testing which were Disk diffusion methods and MIC reduction methods. Two methods designed for the detection of ESBL production Ceftazidime and Ceftazidime plus Clavulinic acid, (CAZ/CAZC) and Cefotaxime and Cefotaxime plus Clavulinic Acid (CTX/CTXC) were used and the PCR was considered as gold standard for evaluation of the other test methods. Result: A total number of 300 GNB were isolated and identified of which $214(71 \%)$ were ESBL positive. For the disk diffusion method, resistant to third generation Cephalosporins were the highest $87.0 \%$, when tested by ceftazidime and by MIC reduction methods were $67 \%$. For the phenotypic confirmatory methods, specificities were $64 \%$ by (CAZ/CAZC), $59 \%$ by (CTX/CTXC) and by both method $52 \%$. Among the phenotypic confirmatory ESBL positive strains by Genotypic method ESBL positive were $50.46 \%$ TEM, $18.69 \%$ SHV and $46.72 \%$ CTX-M gene. Conclusion: Two-step strategies using both DDDT phenotypic methods are useful diagnostic tools for the detection of ESBL from
\end{abstract}

the Gram negative bacilli.

Keywords Extended-Spectrum B-Lactamase-Producing, ESBL, Phenotypic Detection, Double Disc Diffusion Test

\section{Introduction}

Antibiotic resistance has been emerged worldwide. It has been reported that the overuse of third generation of cephalosporins has been associated with the emergence of $\beta$-Lactamases ${ }^{1}$. These enzymes have serine at their active site and attack the amide bond in the $\beta$ lactam ring of amtibiotics $^{2}$. ESBLs are enzymes that mediate resistance to extended spectrum cephalosporins as well as monobactams ${ }^{3}$.

There are no definite guidelines for the detection of extended-spectrum beta-lactamase (ESBL) among Gram negative bacilli especially Enterobacteriaceae as well as Pseudomonas species. Though these species may cause hospital associated infection (HAI) $)^{4-6}$ these are developed frequently multidrug resistant (MDR) ${ }^{5-6}$. These bacteria may constitute a reservoir for plasmid-mediated ESBLs transmission for other Enterobacteriaceae species ${ }^{7-9}$. Phenotypic detection of ESBLs in members of the Enterobacteriaceae co-expressing an AmpC beta-lactamase is complex as because AmpC expression may mask the synergy required for ESBL detection between third-generation cephalosporin and clavulanic acid. This problem may be circumvented by detection of ESBL by molecular technique with specific primer ${ }^{10}$. Most guidelines 
recommend screening isolates based on decreased susceptibility to extended-spectrum cephalosporin in primary susceptibility testing followed by confirmation of ESBL production. However, still there is a controversy about the confirmation of $\mathrm{ESBL}^{11}$. Therefore, detection of ESBL-producing organisms has become a concern for general hospitals and private practice laboratories. The current changes in clinical MIC breakpoints for extended-spectrum cephalosporins and for aztreonam against Enterobacteriaceae by EUCAST and CLSI decrease the likelihood of interpreting an ESBL-producing Enterobacteriaceae as susceptible to extended-spectrum cephalosporins ${ }^{12-14}$. Several phenotypic methods have been developed to confirm ESBL production by Enterobacteriaceae ${ }^{15-17}$. Therefore the purpose of the present study was to compare two phenotypic methods for the detection of ESBL-positive Enterobacteriaceae and Pseudomonas species.

\section{Methodology}

This cross sectional study was carried out in the Department of Microbiology at Mymensingh Medical College, Mymensingh, Bangladesh from January 2011 to June 2011. Mymensingh is $200 \mathrm{KM}$ away from the capital of Bangladesh which is the largest district of this country and Mymensingh Medical College is the only teaching hospital with more than 500 beds with emergency as well as OPD facilities. Patients presented with UTI or wound infection were at any age with both sexes were taken as study population. Non-repetitive clinical isolates were collected from MMCH both the outpatients and inpatients department over a period of 6 months. Urine and wound infection were used as specimen. Laboratory work was carried out in the department of Microbiology in Mymensingh Medical College, Bangladesh. Specimens were collected aseptically ${ }^{18}$. All samples were routinely cultured on MacConkey and blood agar plates at $37^{\circ} \mathrm{C}$ aerobically for 18 hours. Gram negative isolates were further characterized by standard biochemical tests ${ }^{19}$.The susceptibility to antibiotics was determined by Kirby Bauer method according to CLSI 2010 protocols for Gram negative panels ${ }^{20}$. E. coli ATCC 25922 was used as control strains. Three diagnostic tests for ESBL production were performed which were screening test for ESBL by disc diffusion test (DDT) and MIC reduction method, confirmatory test by double disc diffusion test (DDDT) and multiplex PCR for TEM, SHV and CTX-M genes. Isolates were screened for ESBL production by using disc diffusion test on Muller Hinton agar according to the $\mathrm{CLSI}^{21}$ recommendationswhere isolates showing inhibition zone size of $\geq 22 \mathrm{~mm}$ with ceftazidime ( $30 \mu \mathrm{g}), \geq 25 \mathrm{~mm}$ with ceftriaxone $(30 \mu \mathrm{g}), \geq 27 \mathrm{~mm}$ with cefotaxime $(30 \mu \mathrm{g}), \geq 27$ $\mathrm{mm}$ with Aztreonam $(30 \mu \mathrm{g})$ were suspected for ESBL production. MIC reduction method $\geq 8 \mu \mathrm{gm} / \mathrm{ml}$ with
Ceftazidime was performed. E. coli ATCC 25922 was used as a negative control. Phenotypic confirmatory test for ESBL producers were done by double disc diffusion test (DDDT) by two methods which were eftazidime and Ceftazidime plus Clavulinic acid (CAZ/CAZC) and Cefotaxime and Cefotaxime plus Clavulinic Acid (CTX/CTXC). Plasmid DNA was isolated from bacterial cells by alkaline lysis method by Medici et $a l^{22}$. Supernatant $1 \mu 1$ was used as template. The PCR primers and cycling conditions used were previously described ${ }^{23-25}$. Primers used for TEM gene were TEM F, TEM R, SHV gene were SHV F, SHV R, CTX-M gene were CTX-MU-1, CTX-MU-2. Amplification was performed in an Thermocycler (Eppendrof, Takara, Japan) in $1.0 \%$ agarose gel and visualized by staining with ethidium bromide $(0.5 \mathrm{mg} / \mathrm{ml})$ running through horizontal electrophoresis. A 100 bp ladder molecular weight marker (Roche, USA) was used to measure the molecular weights of amplified products. The images of ethidium bromide stained DNA bands were digitized using a gel documentation system (AlphaimagerTM 3400, USA) by UV light. The qualitative data were expressed as frequency and percentage. The comparisons of qualitative data were performed by Chi-square test if data were normally distributed. Statistical analysis was performed by using window based computer software devised with Statistical Packages for Social Sciences (SPSS-17) (SPSS Inc, Chicago, IL, USA). 95\% confidence limit was taken. The summarized data was interpreted accordingly and was then presented in the form of tables. P value less than 0.05 was taken as level of significance.

\section{Result}

A total of 300 bacterial isolates were yielded. The specimens were urine $216(72 \%)$, wound swab $45(15 \%)$ pus $39(13 \%)$. Out of 300 Gram negative isolates in this study majority were Escherichia coli (52\%) followed by Proteus species (18.3\%), Klebsiella species (15\%), Pseudomonas species (3\%) and others (11.7\%). Among 300 GNB 261(87\%) were resistant to Ceftazidime, 242(81\%) were resistant to Ceftriaxone, 191(64\%) were resistant to Cefotaxime and $100 \%$ were in aztreonam (Table 1). In MIC reduction method ( $\geq 8 \mu \mathrm{gm} / \mathrm{ml}$ ) Ceftazidime was resistant in $67 \%$ cases. Most of the bacteria showed ESBL positive by both combination (CAZ/CAZC, and CTX/CTXC). Escherichia coli and Klebsiella species showed maximum ESBLs production in CAZ/CAZC combination. Both the CAZ/CAZC and CTX/CTXC methods were statistically significant. The distribution of ESBLs gene in different isolates rate of TEM, SHV and CTX-M genes present in study population were $50.46 \%, 18.69 \%$ and $46.72 \%$ respectively. 
Table 1. Third generation Cephalosporin Resistant Isolates

\begin{tabular}{ccc}
\hline Name of antibiotic & Frequency & Percentage \\
\hline Ceftazidime & 261 & 87 \\
Ceftriaxone & 242 & 81 \\
Cefotaxime & 191 & 64 \\
Aztrionam & 300 & 100 \\
MIC $(\geq 8 \mu \mathrm{gm})$ & 202 & 67 \\
\hline
\end{tabular}

Table 2. Detection of ESBL by Double Disc Diffusion test as confirmatory test

\begin{tabular}{cccc}
\hline Bacteria Name & CAZ/CAZC & CTX/CTXC & Both \\
\hline Esch. coli $\mathrm{n}=105$ & $23(21.5)$ & $13(12.3)$ & $69(65.7)$ \\
Klebsiella spp. $\mathrm{n}=36$ & $5(13.8)$ & $3(8.3)$ & $28(77.7)$ \\
Proteus spp. $\mathrm{n}=40$ & $5(12.5)$ & $2(5.0)$ & $33(82.5)$ \\
Pseudomonas spp. $\mathrm{n}=8$ & $1(12.5)$ & $1(12.5)$ & $6(75.0)$ \\
*Others $\mathrm{n}=25$ & $4(16.0)$ & $2(8.0)$ & $19(76.0)$ \\
Total $\mathrm{n}=214$ & $38(17.7)$ & $21(9.8)$ & $155(72.4)$ \\
\hline
\end{tabular}

*Others $=$ Enterobacter spp., Citrobacter spp.; Figure within the parenthesis indicates the percentage.

Table 3. Cross tabulation between CAZ/CAZC and CTX/CTXC of ESBL detection

\begin{tabular}{cccc}
\hline ESBL & CAZ/CAZC & Combined & CTX/CTXC \\
\hline Positive & 193 & 155 & 176 \\
Negative & 21 & 59 & 38 \\
Total & 214 & 214 & 214 \\
\hline
\end{tabular}

$*^{2}$ value 22.1 at df $3, p<0.001$, highly significant; $x^{2}$ value 5.8 at df $3, p<0.01$, highly significant; Note: Figures in parentheses represent percentage; Others - Enterobacter spp., Citrobacter spp

Table 4. Pattern of distribution of TEM, SHV and CTX-M genes among phenotypic confirmed ESBL producers

\begin{tabular}{ccc}
\hline Name of Gene & Frequency & Percentage \\
\hline TEM & 54 & 50.46 \\
SHV & 20 & 18.69 \\
CTX & 50 & 46.72 \\
\hline
\end{tabular}

\section{Discussion}

Detection of ESBLs are a great challenge in the clinical microbiology laboratory ${ }^{21-25}$. The CLSI guidelines regarding the sensitivity breakpoints designated for Klebsiella species and Escherichia coli against cefotaxime, ceftriaxone, and ceftazidime are inefficient for the detection of ESBLs ${ }^{26-27}$. Failure to detect ESBL production by routine disc-diffusion tests has been well documented ${ }^{26}$. Highly informative studies were performed by Tenover et $a l^{26}$ in the United States and Livermore $e t a l^{28}$ in Europe and were reported that ESBL mediated resistance are frequently encountered difficulties with both automated and disk diffusion methods. This might be due to the variable affinity of enzymes for different substrates and inoculum effect ${ }^{29}$. Furthermore, ESBL-producing organism gives the treatment failure with third generation cephalosporin if the infection is outside the urinary $\operatorname{tract}^{27}$. In addition to that reports of failures in both animal models and clinical settings are well documented when third-generation cephalosporins are used to treat ESBL infections $^{30-31}$ The sensitivity of any particular extended-spectrum cephalosporin used as a screening test can be dependent on the geographic variation and the resistance patterns of these organisms ${ }^{20}$. More resistant to ceftazidime is reported and SHV-3 like ESBLs has been found which is $100.0 \%$ resistant to ceftriaxone; whereas only $50.0 \%$ are resistant to ceftazidime ${ }^{30}$. In this study resistant to Ceftazidime is found in $87.0 \%$. Moland et $a l^{31}$ has used ceftazidime resistance as marker for ESBL production which is $78.0 \%$ in a broth dilution format. Similarly it has been found statistically more significant by these combinations. Though ceftazidime plus clavulinic acid (CAZ/CAZC) is the best single disc diffusion test for the detection of $\mathrm{ESBL}^{25}$, it cannot cover all the strain. Use of only one combination may fail to detect ESBL positive strains; thus this might give underestimation of prevalence of ESBL. In this study both combinations were found statistically significant. The use of cefotaxime, ceftazidime and ceftriaxone as the only indicator of ESBLs screening can no longer be recommended. If only one indicator antibiotic would be used for screening, 
cefpodoxime has proven to be the best molecule for screening all types ESBLs producers in clinical sample and this also produces AmpC $\beta$-lactamase ${ }^{23}$. In this study two combinations with clavulanic acid like CAZ/CAZC and $\mathrm{CTX/CTXC}$ are tested and have been found that $E$. coli and Klebsiella species showed maximum ESBLs production in CAZ/CAZC combination which correlates with other studies $^{31}$. However, the combination of ceftazidime and cefotaxime still missed two strains producing the SHV-5 and SHV-7 ESBLs ${ }^{30}$. If the both combination does not use the actual figure may not appear and may show low prevalence rate. These types of discrepancies between susceptibility data and disc diffusion results have increased the need for an improved method of ESBL detection which can be incorporated into the routine susceptibility procedure.

This study should be viewed in the context of several limitations. Firstly, because of constraints of time and budget, this was a single centered with relatively small sample size; therefore, the results cannot be generalized to the whole country. Furthermore, antibiotic history of the patients before and after in vitro sensitivity test was not considered and only in vitro drug response was taken as the result. PCR-based molecular assays have their own limitations. These need expensive equipment and reagents and expert personnel which are not always available to the diagnostic laboratory. In addition, primers have to be designed within low mutation rate regions and the use of specific primers hinders the identification of novel resistance genes giving false negative results. However, so far this type of study has not been performed in Bangladesh. Further study is needed to correlate between in vitro and in vivo drug response among the patients infected with ESBLs producing bacteria.

\section{Conclusions}

In conclusion routine used of both DDDT combinations is useful for the detection of ESBL in clinical samples. This is a better method for the detection of ESBL producing bacteria especially enterobacteriaceae and Pseudomonas species.

\section{REFERENCES}

[1] Andrews J, 2009, Detection of extended spectrum $\beta$ lactamases (ESBLs) in E.coli and Klebsiella species. British society for antimicrobial chemotherapy; 674-675

[2] Chaudhary U, Aggarwal R, 2004, Extended spectrum beta lactamases (ESBL) An emerging threat to clinical therapeutics, Indian Journal of Medical Microbiology ; 22 (2): $75-80$

[3] Paterson D L, Bonomo R A, 2005,Extended-spectrum beta-lactamases: a clinical update, Clin Microbiol Review; 18(4): 657-86

[4] Manzur A., et al 2007. Nosocomial outbreak due to extended-spectrum-beta-lactamase-producing Enterobacter cloacae in a cardiothoracic intensive care unit. J. Clin. Microbiol. 45:2365-2369

[5] Paauw A., Fluit A. C., Verhoef J., Leverstein-van Hall M. A.2006. Enterobacter cloacae outbreak and emergence of quinolone resistance gene in Dutch hospital. Emerg. Infect. Dis. 12:807-812

[6] Potron A., et al2009. Nosocomial spread of ESBL-positive Enterobacter cloacae co-expressing plasmid-mediated quinolone resistance Qnr determinants in one hospital in France. J. Antimicrob. Chemother. 64:653-654

[7] Crowley B., Ratcliffe G.2003. Extended-spectrum beta-lactamases in Enterobacter cloacae: underestimated but clinically significant! J. Antimicrob. Chemother. 51:1316-1317

[8] Szabó D., et al2005. SHV-type extended-spectrum beta-lactamase production is associated with reduced cefepime susceptibility in Enterobacter cloacae. J. Clin. Microbiol. 43:5058-5064

[9] Towne T. G., Lewis J. S., Herrera M., Wickes B., Jorgensen J. H.2010. Detection of SHV-type extended-spectrum beta-lactamase in Enterobacter isolates. J. Clin. Microbiol. 48:298-299

[10] James Cohen Stuart'Bram Diederen' Nashwan al Naiemi' Ad Fluit' Niek Arents'Steven Thijsen' Bart Vlaminckx' Johan W. Mouton and Maurine Leverstein-van Hall,2011,Method for Phenotypic Detection of Extended-Spectrum Beta-Lactamases in Enterobacter Species in the Routine Clinical Setting, J. Clin. Microbiol. vol. 49 no. 7 2711-2713

[11] Hélène Garrec, Laurence Drieux-Rouzet, Jean-Louis Golmard, Vincent Jarlier, and Jérôme Robert,2011,Comparison of Nine Phenotypic Methods for Detection of Extended-Spectrum $\beta$-Lactamase Production by Enterobacteriaceae vol.49 no.3 1048-1057.

[12] Andrea Bartolini, Ilaria Frasson, Antonietta Cavallaro, Sara N Richter and Giorgio Palù' 2014,Comparison of phenotypic methods for the detection of carbapenem non-susceptible EnterobacteriaceaeGut Pathogens2014, 6:13

[13] Cheessbrough M, 2006, District laboratory practice in tropical countries. Microbiology -2 , New york, USA : Cambridge university; 184-186

[14] Clinical Laboratory Standards Institute. Performance standards for antimicrobial discsusceptibility testing. 14th informational supplement. 2010

[15] Clinical Laboratory Standards Institute. Performance standards for antimicrobial susceptibility testing. 16th informational supplement .2006; M100-S15

[16] Medici DD, Croci L, Delibato E, Pasquale SD, Filetici E and Toti L, 2003, Evaluation of DNA Extraction Methods for Use in Combination with SYBR Green I Real-Time PCR To Detect Salmonella enterica Serotype Enteritidis in Poultry, Applied and Environmental Microbiology, vol. 69, no. 6, pp. 3456-3461

[17] Pagani L, Amico ED, Migliavacca R, D’Andrea MM, Giacobone E, Amicosante G, Romero E, and Rossolini GM, 2003, Multiple CTX-M-Type Extended-Spectrum $\beta$-Lactamases in Nosocomial Isolates of Enterobacteriaceae from a Hospital in Northern Italy, Journal of Clinical 
Microbiology, vol. 41, no. 9, pp. 4264-4269

[18] Chia J, Chu C, Su L, Chiu C, Kuo A, Sun C and Wul T, 2005, Development of a Multiplex PCR and SHV Melting-Curve Mutation Detection System for Detection of Some SHV and CTX-M $\beta$-Lactamases of Escherichia coli, Klebsiella pneumoniae, and Enterobacter cloacae in Taiwan, Journal of Clinical Microbiology, pp- 4486-4491

[19] Lal P, Kapil A, Das B K and Sood S, 2007, Occurence of TEM and SHV gene in extended spectrum beta lactamases (ESBLs) producing Klebsiella spp.isolated from a tertiary care hospital, Indian Journal Medcal Research; 125: $173-178$

[20] Tenover FC, Mohammed MJ, Gorton TS, Dembek ZF (1999) Detection and reporting of organisms producing extended-spectrum ß-Lactamase: survey of laboratories in Connecticut. J Clin Microbiol 37: 4065-70

[21] Steward CD, Wallace D, Hubert SK, Lawton R, Fridkin SK, et al. (2000) Ability of laboratories to detect emerging antimicrobial resistance in nosocomial pathogens: a survey of Project ICARE laboratories. Diagn Microbiol Infect Dis 38: 59-67

[22] Livermore DM, Hawkey PM (2008) CTX-M: changing the face of ESBLs in the UK. 2005, defining an extended-spectrum beta-lactamase. Clin Microbiol Infect5: 21-24

[23] Karas JA, Pillay, DG, Muckart D, Sturm AW (1996) Treatment failure due to extended spectrum B-Lactamase. J Antimicrob Chemother 37: 203-204

[24] Ensor VM, Shahid M, Evans JT, Hawkey PM (2006) Occurrence, prevalence and genetic environment of CTX-M b-lactamases in Enterobacteriaceae from Indian hospitals. J Antimicrob Chemother 58: 1260-1263

[25] Peirano G, Pitout JDD (2010) Molecular epidemiology of Escherichia coli producing CTX-M $\beta$ Lactamases: worldwide emergence of clone ST131 025:H4. Int J Antimicrob Agents35: 316-321

[26] Cavaco LM, Abatih E, Aarestrup FM, Guardabassi L (2008) Selection and Persistence of CTX-M producing Esch. Coli in the intestinal Flora of Pigs Treated with Amoxicillin, Ceftiofur or Cefquinome. Antimicrob Agents Chemothr 52 (10): 3612-3616

[27] Farkosh MS, Extended-Spectrum beta lactamase Producing Gram Negative Bacilli. 2007; http://nosoweb.org/infectious diseases/esbl.htm (accessed on 5 April, 2011)

[28] Moland ES, Black JA, Ourada J, Reisbig MD, HansonND, et al.(2002)Occurrence of newer beta-lactamases in Klebsiella pneumoniae isolates from 24 U.S. hospitals. Antimicrob Agents Chemother 46: 3837-3842

[29] George A, Jacoby MD, Silvia Munoz-Price LS (2005) "mechanisms of disease: The New beta-Lactamases." N Engl J Med35:380-391

[30] Rahman MM, Haque JA, Hossain MA, et al. (2004) Prevalence of extended spectrum beta lactamase-producing Escheria coli and Klebsiella pneumoniae in an urban hospital in Dhaka Bangladesh. Int J Antimicrob Agents 24 (5): $508-510$

[31] Black JA, Thomson KS, Buynak JD, Pitout JD (2005) Evaluation of $\beta$-lactamase inhibitors in disc tests for detection of plasmid-mediated AmpC $\beta$ lactamases in well characterized clinical strains of Klebsiella spp. J Clin Microbiol 43: 4161-4171 